\title{
ERGONOMÍA EN LOS SISTEMAS DE SALUD DE AMÉRICA LATINA: REVISIÓN SISTEMÁTICA DE LA SITUACIÓN ACTUAL, NECESIDADES Y DESAFÍOS FUTUROS
}

\author{
HUMAN FACTORS/ERGONOMICS IN LATIN AMERICAN HEALTH SYSTEMS: \\ SYSTEMATIC REVIEW OF CURRENT STATUS, NEEDS AND FUTURE CHALLENGES
}

\author{
Carlos Aceves-González* \\ Irma Cecilia Landa-Ávila ${ }^{* *}$ \\ Fernando Carvalho*** \\ Bertha Alicia Ortega-Ruiz ${ }^{* * *}$ \\ Gyuchan Thomas Jun ${ }^{* * * *}$
}

\begin{abstract}
Resumen: La ergonomía (o factores humanos) (E/FH) es una disciplina científica que ayuda a mejorar, (re)diseñar e implementar sistemas de salud resilientes y seguros que garanticen la seguridad de los pacientes y el bienestar de todos los actores involucrados. A pesar de los beneficios de integrar la E/FH en los sistemas de salud, su diseminación y aplicación en América Latina ha sido lenta. Este artículo identifica los aportes en investigación de la E/FH a los sistemas de salud de América Latina a través de una revisión sistemática de literatura. La revisión siguió las directrices PRISMA, realizando la búsqueda en inglés, español y portugués en las bases de datos Scopus, Web of Science, PubMed y Scielo. Un total de 77 artículos fueron incluidos en la revisión y analizados acorde con los cinco dominios en que se ha enfocado la E/FH en los sistemas de salud. Estos dominios incluyen: i) usabilidad de la tecnología, ii) seguridad del paciente y análisis de incidentes, iii) desempeño y seguridad de los trabajadores, iv) diseño de sistemas, y v) resiliencia de sistemas. La mayoría de los estudios se han centrado en el desempeño y seguridad de los trabajadores y la usabilidad de la tecnología; mientras que los otros tres dominios han sido menos estudiados. Lo que sugiere la necesidad de incentivar el uso de los dominios de E/FH con un enfoque de diseño de sistemas, resiliencia de sistemas y de análisis de incidentes que han demostrado ser más eficaces para mejorar los sistemas de salud.
\end{abstract}

Palabras clave: Factores humanos, ergonomía y cuidado de la salud, América Latina, seguridad del paciente, calidad, revisión sistemática.

Abstract: Ergonomics (or human factors) (E/FH) is a scientific discipline that helps to improve, (re)design and implement resilient and safe health systems that ensure patient safety and all

"Universidad de Guadalajara. Guadalajara, México. Correo electrónico: c.aceves@academicos.udg.mx. Orcid: https://orcid.org/o0oo-0002-6720-808X. Autor de correspondencia.

${ }^{* *}$ Loughborough University. Loughborough, Reino Unido. Correo electrónico: i.c.landaavila@lboro.ac.uk. Orcid: https://orcid.org/oooo-0001-6107-6736

***Nottingham Trent University. Nottingham, Reino Unido. Correo electrónico: designandbehaviour@gmail.com. Orcid: https://orcid.org/oooo-0oo2-4736-456o

*****Universidad de Guadalajara. Guadalajara, México. Correo electrónico: bertha.ortega@alumnos.udg.mx. Orcid: https://orcid.org/oooo-0002-5468-7878

******Loughborough University. Loughborough, Reino Unido. Correo electrónico: G.Jun@lboro.ac.uk. Orcid: https://orcid.org/0000-0002-0958-0107 
stakeholders' well-being. Despite the benefits of integrating $\mathrm{E} / \mathrm{FH}$ into health systems, its dissemination and application in Latin America have been slow. This article identifies the research contributions of E/FH to Latin American health systems through a systematic literature review. The review followed the PRISMA guidelines, searching in English, Spanish and Portuguese in the Scopus, Web of Science, PubMed and Scielo databases. A total of 77 papers were included in the review and analysed according to the five domains in which $\mathrm{E} / \mathrm{FH}$ has focused on health systems. These domains include: i) technology usability, ii) patient safety and incident analysis, iii) worker performance and safety, iv) system design, and v) system resilience. Most studies have focused on worker performance and safety, and technology usability, while the other three domains have been less studied. This suggests the need to incentivise the E/FH domains, focusing on systems design, systems resilience, and incident analysis that have shown to be most effective in improving health systems.

Keywords: Human factors, healthcare ergonomics, Latin America, patient safety, quality of care, systematic review.

Recepción: 15.07.2021 / Revisión: 30.07.2021 / Aceptación: 03.08.2021

\section{Introducción}

Los sistemas de salud en Latinoamérica enfrentan múltiples presiones, como inequidad en el acceso, cuestionable calidad en el servicio, falta de infraestructura y recursos insuficientes lo que genera bajos niveles de cobertura (Organisation for Economic Cooperation and Development [OECD]/The World Bank, 2020), además de los problemas asociados con la seguridad del paciente (World Health Organization [WHO], 2011). Para resolver algunos de estos problemas, existe la oportunidad de integrar disciplinas menos exploradas como el caso de la ergonomía, también llamada factores humanos (E/FH). A nivel internacional la Organización Mundial de la Salud (OMS) ha señalado su valor para mejorar los sistemas de salud (WHO, 2021), y se ha reconocido como una disciplina que ayuda en áreas específicas como uso seguro de medicamentos, la implementación de nuevas tecnologías y la seguridad del paciente (Carayon et al., 2014b). La E/FH es una disciplina científica que se enfoca en comprender la complejidad de los sistemas de trabajo y en cómo la interacción entre las personas y los elementos de un sistema puede influir en el desempeño del mismo y el bienestar de todos los actores involucrados (Dul et al., 2012; International Ergonomics Association [IEA], 2000).

A pesar del reconocimiento de la E/FH como disciplina para mejorar los sistemas de salud, el nivel de progreso ha sido diferente en las distintas partes del mundo, y existe una comprensión limitada de las contribuciones que la $\mathrm{E} / \mathrm{FH}$ ha hecho a los sistemas de salud en América Latina (Aceves-González et al., 2021). Por ende, es indispensable desarrollar una visión más amplia sobre las aplicaciones existentes de la Ergonomía en el cuidado de la salud en América Latina e identificar las brechas y las oportunidades futuras de investigación y su aplicación.

Este articulo tiene como objetivo identificar, a través de una revisión sistemática, los aportes en investigación de la E/FH a los sistemas de salud de América Latina y cuáles son las áreas de oportunidad para aumentar su diseminación y aplicación en la región. Este 
artículo primero presenta una descripción de la E/FH en el contexto de la salud y sus dominios de aplicación, seguido de la metodología de la revisión sistemática y los resultados; finalmente, concluye con la discusión sobre los dominios de aplicación y las posibles implicaciones.

\section{Aplicación de la ergonomía en los sistemas de salud}

El conocimiento y la experiencia de la ergonomía/factores humanos (E/FH) ha surgido principalmente en su aplicación en productos de consumo y software, o en industrias con aplicación de ingeniería de alto nivel como la industria nuclear o de aviación (Waterson, 2011). Aunque existen estudios pioneros, como los desarrollados por Chapanis y Safren en materia de uso seguro de medicamentos (Chapanis \& Safren, 1960; Safren \& Chapanis, 1960), la aplicación de la ergonomía en el sector de la salud es relativamente nueva.

La literatura en el área indica que en las décadas de los 80’s y 90s, la contribución de la ergonomía se centró en la salud ocupacional del personal hospitalario, el diseño de clínicas y hospitales y en algunos esfuerzos para incorporar el enfoque de sistemas como parte de la cultura organizacional de la atención médica (Chartered Institute of Ergonomics and Human Factors [CIEHF], 2018). Sin embargo, con la publicación del informe "To Err is Human: Building a Safer Health System" (Kohn et al., 2000) la ergonomía y su enfoque de sistemas fue reconocido como elemento crítico para la seguridad del paciente en todos los dominios de atención médica (Carayon et al., 2014a). En las últimas dos décadas, y principalmente en los países de altos ingresos, una gran cantidad de personas y sistemas de salud se han visto beneficiados con la integración de esta disciplina en diversas áreas de la atención médica, como cuidados intensivos, atención, emergencias, así como en el diseño de dispositivos médicos, el entrenamiento, la mejora de la calidad, la seguridad, la comunicación entre equipos de trabajo, la orientación a la comunidad, la gestión de riesgos, los aspectos de la cultura organizacional, la carga de trabajo, y en general la confiabilidad en los sistemas de salud (Catchpole et al., 2021; Hignett et al., 2013).

Sin embargo, el avance para integrar la $\mathrm{E} / \mathrm{FH}$ en los países de bajos y medianos ingresos ha sido más lento, a pesar de que existe una gran urgencia de su aplicación dado que las necesidades y las desigualdades son mayores que en otras áreas del mundo (AcevesGonzález et al., 2021; Scott, 2008). De acuerdo con Thatcher y Todd (2019) la aplicación de la ergonomía en América Latina ha sucedido, principalmente, en materia de salud ocupacional en el sector de la manufactura, con menos aplicaciones en el sector de servicios. AcevesGonzález et al. (2021) señalan que, en cuanto a sistemas de salud, en América Latina existe una mayor necesidad de integrar la $\mathrm{E} / \mathrm{FH}$ que en los países de mayor ingreso. No obstante, existe al mismo tiempo desconocimiento por parte de los actores involucrados sobre el potencial de esta disciplina, por tanto, su aplicación es limitada.

Un aspecto de alta relevancia para la integración de la ergonomía y los sistemas de salud, es el hecho de que recientemente la Organización Mundial para la salud (OMS), en su Plan de Acción Global para la Seguridad del Paciente 2021-2030 (WHO, 2021), ha señalado a la E/FH como una estrategia clave para el construir sistemas y organizaciones de salud de alta confiabilidad que protejan a los pacientes de daños que ocurren a diario. Incluso, cabe destacar que en medio de la pandemia por la COVID-19, la OMS ha puesto énfasis no solo en 
la seguridad del paciente, sino también en el bienestar de los trabajadores de la salud; para ambos casos, la ergonomía ha sido reconocida tanto para mejorar la seguridad de los pacientes, como el bienestar de todos los actores involucrados (Carayon et al., 2014b).

Dominios de aplicación de la ergonomía en los sistemas de salud

Debido al amplio alcance de la E/FH, su integración en los sistemas de salud puede darse en distintos dominios. Carayon et al. (2014b) han identificado cinco dominios principales en los que la $\mathrm{E} / \mathrm{FH}$ puede contribuir en los sistemas de salud:

1) Usabilidad de la tecnología: Este dominio se enfoca al diseño de dispositivos médicos utilizables y seguros y el desarrollo de tecnología de la información sanitaria.

2) Análisis de incidentes de seguridad del paciente: Comprender la naturaleza del error humano y los factores subyacentes que contribuyen a los incidentes de seguridad del paciente.

3) Desempeño y seguridad de los trabajadores de la salud: Identificación de obstáculos de desempeño (físicos, cognitivos y sociales/conductuales) que enfrentan los trabajadores de la salud.

4) Diseño de sistemas: Aplicación de un enfoque de sistemas para diseñar sistemas integrales de atención al paciente y múltiples interacciones. Es relevante resaltar que la aplicación la ergonomía implica el uso de un enfoque de sistemas (Wilson, 2014).

5) Resiliencia organizacional/del sistema: Mejora de la capacidad del sistema/ organización para anticiparse y adaptarse al potencial de sorpresa y falla.

\section{Materiales y métodos}

Esta revisión tuvo como objetivo explorar los cinco dominios de investigación E/FH enfocadas en América Latina. Para ello, se realizó una revisión sistemática de publicaciones académicas siguiendo las directrices PRISMA. El proceso se resume en los siguientes cinco pasos:

1) Definición de palabras clave. Los términos primarios Ergonomics, Healthcare y Latinamerica fueron identificados para la búsqueda; a partir de ellas, se determinaron palabras alternativas. La búsqueda se realizó con los términos en inglés, español y portugués (tabla 1). Para evitar los artículos no relevantes, la búsqueda se realizó utilizando el operador booleano "AND" entre los términos primarios.

2) Identificación de artículos mediante búsqueda en bases de datos PubMed, Web of Science, Scopus y Scielo. En las primeras tres bases de datos la búsqueda se realizó en inglés, seguido de una búsqueda en español y portugués en la base de datos de Scielo. Este proceso fue realizado entre el 25 y 28 de febrero de 2021.

3) Aplicación los criterios de inclusión que consistieron en: i) los artículos podían estar enfocados a cualquier nivel/área del sistema de salud, ii) los artículos debían describir estudios originales, iii) la recolección de datos debió realizarse en un país de América 
Latina, y iv) artículos que discuten más de una disciplina fueron incluidos, siempre que la ergonomía fuera una parte esencial del estudio. En este paso no se impusieron restricciones en el año de publicación, y se incluyeron artículos de revistas y conferencias.

4) Rondas de revisiones para determinar lista de artículos incluidos. Los artículos identificados se importaron a la plataforma COVIDENCE para ser revisados por al menos dos de los cinco investigadores. La primera revisión fue sobre el título, resumen y palabras clave; para pasar a la siguiente revisión de texto completo. En este paso se decidió que los artículos de conferencia no serían incluidos.

5) Extracción de información de artículos seleccionados a partir de definir el formato de extracción que incluyó los cinco dominios en los que E/FH ha contribuido en los sistemas de salud (Carayon et al., 2014b). Estos dominios no se excluyen mutuamente y un artículo se puede clasificar en más de uno de ellos.

Tabla 1. Términos clave utilizados para la revisión sistemática.

\begin{tabular}{|c|c|}
\hline Términos primarios de búsqueda & Términos alternativos de búsqueda \\
\hline Ergonomía (Ergonomics) & $\begin{array}{l}\text { OR "factores humanos" OR "ingeniería de resiliencia" } \\
\text { OR "usabilidad de diseño" }\end{array}$ \\
\hline Salud (Healthcare) & $\begin{array}{l}\text { OR "seguridad del paciente" OR "mejora de la } \\
\text { calidad" OR "experiencia del paciente" OR "Diseño } \\
\text { del cuidado de la salud" OR "calidad en el cuidado de } \\
\text { la salud" OR "sistemas de salud" }\end{array}$ \\
\hline América Latina (Latinamerica) & $\begin{array}{l}\text { OR “América del Sur” OR “América Central” OR } \\
\text { Argentina OR Bolivia OR Brasil OR Chile OR } \\
\text { Colombia OR “Costa Rica” OR Cuba OR México OR } \\
\text { Paraguay OR Perú OR Uruguay OR Ecuador OR } \\
\text { Venezuela OR “Indias Occidentales” }\end{array}$ \\
\hline
\end{tabular}

\section{Resultados y discusión}

Resultados de la búsqueda y características de los artículos incluidos en la revisión

Se identificaron inicialmente un total de 1.353 artículos en las bases de datos consultadas (827 en Scopus, 58 en Web of Science, 147 en Pubmed, 321 en Scielo - 118 en español y 203 en portugués-), mismos que se redujeron a 1.299 después de eliminar aquellos que estaban duplicados. Después de examinar el título, el resumen y las palabras clave de cada artículo, se identificaron 303 artículos que fueron considerados para su revisión a texto completo, en ese proceso 77 artículos fueron incluidos en la revisión con base en los criterios de inclusión. La figura 1 resume el proceso para definir los artículos a incluir, siguiendo la estrategia de búsqueda PRISMA.

La tabla A1 (ver anexo) enlista los artículos seleccionados en la revisión. Los estudios están ordenados de acuerdo al dominio de aplicación; se presenta el autor(es), nombre de la revista y disciplina, población de estudio y palabra asociada con $\mathrm{E} / \mathrm{FH}$. 
Figura 1. Diagrama PRISMA.

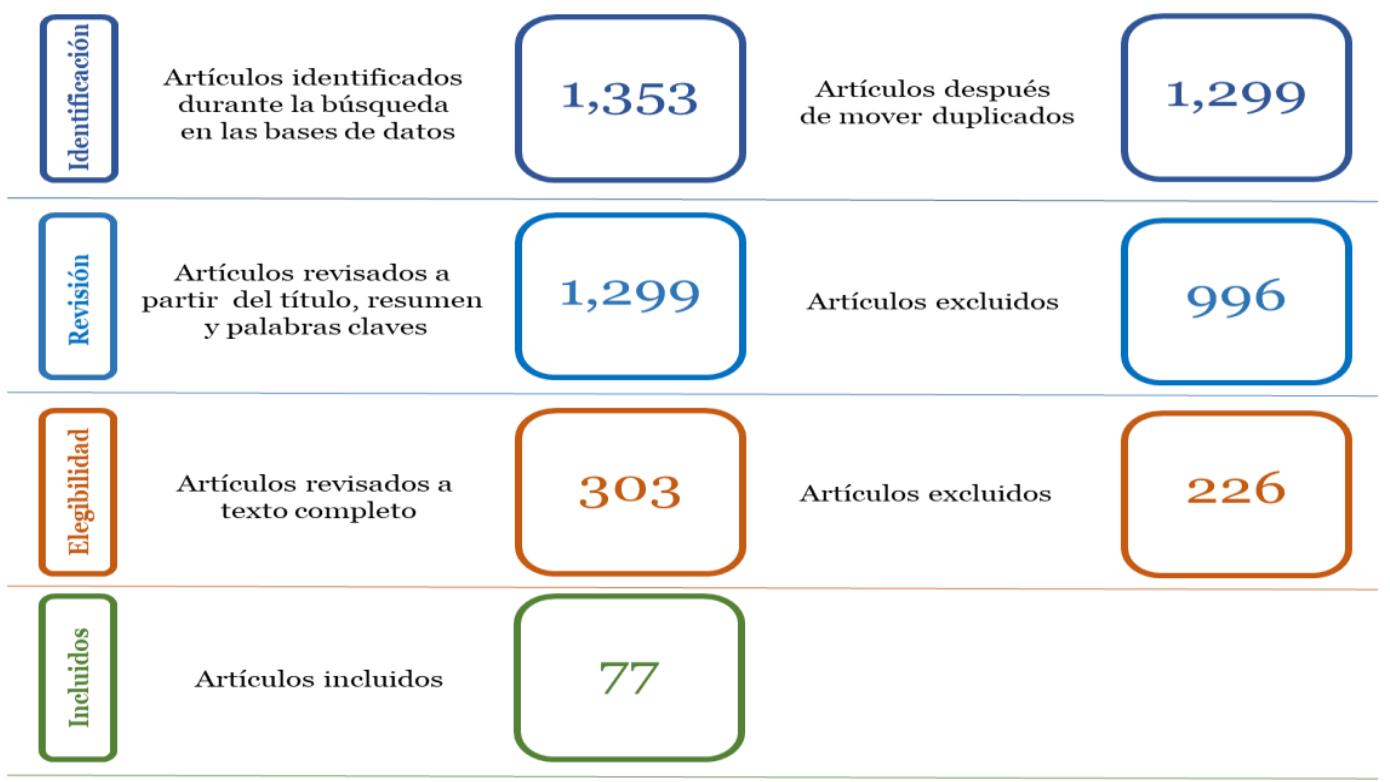

Los estudios seleccionados fueron realizados en varios países, con un claro predominio de las investigaciones realizadas en Brasil (64 estudios), y el resto en Colombia (3 estudios), Argentina, Perú y México (2 estudios por país), y en Venezuela, Cuba, Chile y Ecuador (un estudio por país). Los datos de publicación muestran estudios pioneros de relevancia en el periodo 2001-2012 (16 estudios), y un fuerte aumento en el número de estudios publicados en el periodo 2013-2021 (61 estudios) (figura 2).

Figura 2. Número total de artículos identificados por año de revisión.

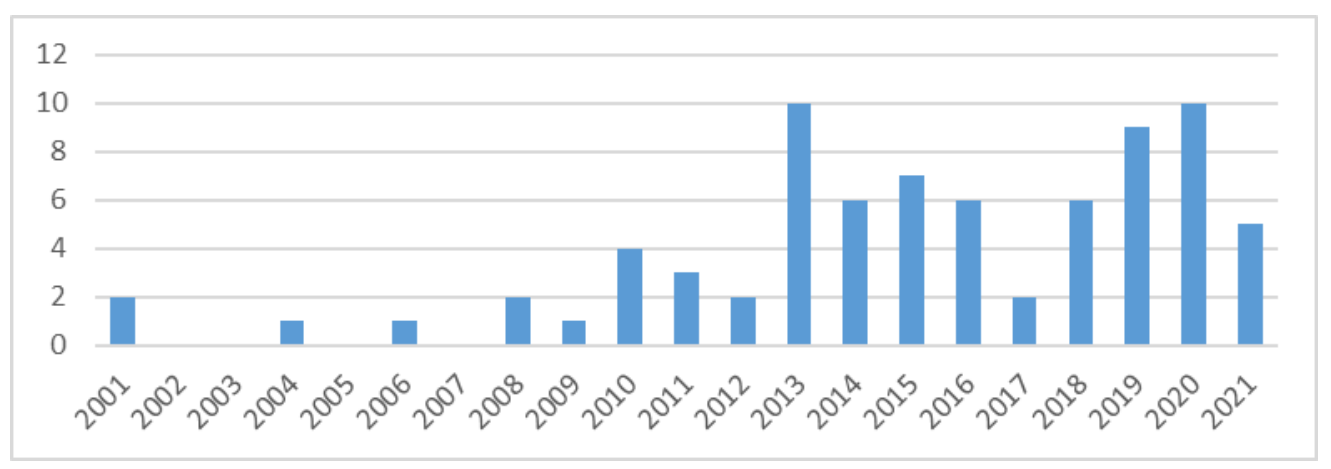

Los artículos fueron publicados en un total de 37 revistas, el 66,3\% (51) de los estudios se publicaron en 21 revistas de América Latina; mientras que el 33,7\% (26) de los artículos fueron publicados en 16 revistas fuera de esta región. La tabla 2 incluye las seis revistas que contienen más artículos incluidos en la revisión. En el lenguaje de las publicaciones, destaca el inglés (30 artículos), portugués (15 artículos) y los artículos publicados en su lengua original (prioritariamente portugués) y además en inglés (24 artículos); solo ocho de los artículos incluidos fueron publicados en español. 
Tabla 2. Revistas con más artículos publicados, incluidos en la revisión.

\begin{tabular}{|l|c|c|}
\hline Nombre revista & Número de artículos (\%) & Idioma \\
\hline Applied Ergonomics & $9(11,7 \%)$ & Inglés \\
\hline Fisioterapia em movimento & $4(5,2 \%)$ & Portugués \\
\hline Revista Latino-Americana de Enfermagem & $4(5,2 \%)$ & Portugués \\
\hline Acta Paulista de Enfermagem & $3(3,9 \%)$ & Portugués \\
\hline Ciencia \& saude coletiva & $3(3,9 \%)$ & Portugués \\
\hline Texto \& Contexto Enfermagem & $3(3,9 \%)$ & Portugués \\
\hline
\end{tabular}

Resultados por dominio de aplicación de la ergonomía en los sistemas de salud

La figura 3 muestra como la mayoría de los estudios incluidos han realizado investigación en el dominio de desempeño y seguridad de los trabajadores (32) y usabilidad de tecnología (26). Los dominios diseño de sistemas (9) y resiliencia organizacional/de sistemas (7) tuvieron menor representación. Tres estudios fueron clasificados en más de un dominio. No se encontró ningún estudio en el dominio de análisis de incidentes de seguridad del paciente.

Figura 3. Número de artículos por dominio de aplicación de la E/FH en los sistemas de salud.

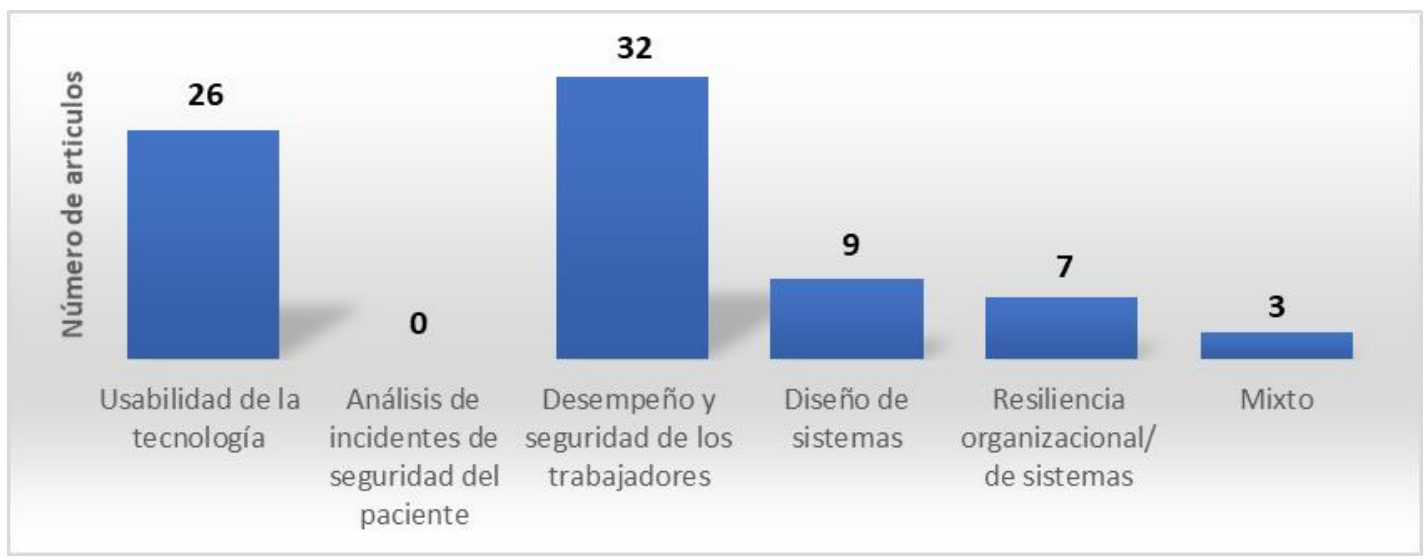

Según la revista de publicación, destacan las disciplinas de enfermería, salud pública, fisioterapia, salud y seguridad ocupacional, educación en salud, ingeniería biomédica, tecnologías de la información, entre otras. Con respecto a los participantes, destacan los estudios con personal de enfermería (28), profesionales de la salud sin especificar profesión (17), médicos (15), profesionales de tecnología de la información (TI) (6), otras profesiones no clínicas (camilleros, conductores de ambulancia, personal de limpieza entre otros) (10), personal administrativo y de apoyo (12) y directivos (1); así como algunos estudios con participación de pacientes (10), usuarios (4) y cuidadores (1). Las palabras más frecuentes asociadas a la E/FH fueron ergonomía (42 estudios), usabilidad (19 estudios) e ingeniería de resiliencia (5 estudios); otras palabras asociadas fueron sistemas complejos, uso de dispositivos, síntomas y molestias musculoesqueléticas, interacción humano-computadora, diseño centrado en el usuario y bienestar humano.

\section{Discusión}

El objetivo de esta revisión sistemática era identificar los aportes en investigación en E/FH a los sistemas de salud de América Latina. La revisión permitió la identificación de estudios que han aplicado la perspectiva de la $\mathrm{E} / \mathrm{FH}$ en diversos dominios y disciplinas del sector 
salud; la mayoría de esos estudios se realizaron en los últimos 10 años, lo cual muestra que la E/FH como tema de investigación está ganando lentamente impulso dentro de este sector. En general, de esta revisión sistemática se desprenden tres hallazgos principales, entre ellos: 1) el predominio de estudios sobre desempeño y seguridad de los trabajadores y usabilidad de la tecnología; 2) la escasez de estudios con una perspectiva de sistemas; y 3) la falta de estudios sobre el análisis de incidentes de seguridad del paciente.

Predominio de estudios sobre seguridad de los trabajadores y usabilidad de la tecnología

Los resultados de esta revisión sugieren que el 42\% (32) de los estudios incluidos están dentro del dominio de desempeño y seguridad de los trabajadores de la salud -también considerado como ergonomía ocupacional o incluso salud ocupacional -. En muchos aspectos, esto era un resultado esperado, ya que es una de las áreas de mayor tradición en el uso de la disciplina (Hignett et al., 2013), y que se ha reproducido en América Latina (Soares, 2006; Thatcher \& Todd, 2019). Usualmente, la ergonomía ocupacional resalta el bienestar de los trabajadores como uno de los resultados y beneficios esperados del uso de la E/FH (Dul et al., 2012; IEA, 2000), lo que ha motivado su diseminación en el sector salud. El porcentaje de estudios en ergonomía ocupacional también responde a los altos índices de trastornos musculoesqueléticos (TMEs) que se registran en la mayoría de los grupos profesionales del personal de salud, con la enfermería como el grupo de mayor riesgo (Hignett et al., 2013), lo cual coincide con los resultados de la revisión en el que los profesionales de enfermería son el grupo de participantes más estudiado. Aunado, los riesgos asociados a las demandas psicológicas y organizacionales del trabajo derivan en situaciones de estrés, ansiedad o burnout, entre otros.

Por otro lado, casi un 40\% (29) de los artículos incluidos en la revisión están dentro del dominio de usabilidad de la tecnología. Los estudios incluyen un amplio rango de herramientas utilizando tecnología de la información (por ejemplo, aplicaciones para dispositivos telefónicos) y algunos dispositivos médicos. El número creciente de estudios en este dominio puede ser explicado por el incremento en la confianza de que las soluciones tecnológicas pueden resolver muchos de los problemas en el sector de la salud (Hignett et al., 2013), aunada a la diseminación de disciplinas como la experiencia de usuario, que enfatizan la importancia de investigar esta área. Es por ello que se cuenta con un mayor número de literatura científica relacionada con problemas de usabilidad en los sistemas de salud (Bitkina et al., 2020).

\section{Escasez de estudios con una perspectiva de sistemas}

Por definición y como parte de sus características fundamentales, la $\mathrm{E} / \mathrm{FH}$ debe integrar un enfoque de sistemas (Dul et al., 2012; IEA, 2000; Wilson, 2014). Mientras que en los dos dominios anteriores se concentra aproximadamente el 80\% de los estudios en E/FH y salud, la mayor parte de estos estudios no utilizan una perspectiva de sistemas para el desarrollo de su investigación. Por ejemplo, entre los estudios de desempeño y seguridad de los trabajadores de la salud es común que se busque identificar la relación entre los riesgos ergonómicos físicos (posturas y manejo de pacientes, por ejemplo) y la presencia de trastornos musculoesqueléticos (TMEs) (Lima et al., 2018; Montalvo-Prieto et al., 2015), pero con menor énfasis en relacionarlos con riesgos o factores cognitivos (carga mental, 
estrés, distractores, entre otros) u organizacionales (comunicación, turnos, carga de trabajo, entre otros) que podrían afectar la salud y el desempeño de los trabajadores. Igualmente, es poco común que en estos estudios se revise cómo los TMEs pueden afectar el desempeño del sistema y causar consecuencias inesperadas con sus resultados, por ejemplo, en términos de seguridad del paciente.

Esto genera que la mayor parte de la investigación tiende a estudiar un número limitado de interacciones de los elementos del sistema. Entre los artículos incluidos en esta revisión, solo un 12\% (9) de los artículos se clasificó dentro del enfoque de diseño sistemas. Esto puede deberse a que la implementación de un enfoque de sistemas es compleja, y aún existe una carencia a nivel internacional sobre enfoques/métodos prácticos y sencillos que ayuden a introducir esta práctica (Chughtai \& Blanchet, 2017). Sin embargo, existe un reconocimiento e interés de la comunidad médica por integrar el enfoque de sistemas para informar su práctica (Churruca et al., 2019; Plack et al., 2018). Otro factor puede estar relacionado con la magnitud de los problemas y los limitados recursos para la investigación, que a menudo limitan el uso de un verdadero enfoque de sistemas (Waterson, 2009).

Otro elemento menos explorado del enfoque de sistemas en la literatura latinoamericana, es la integración de múltiples actores involucrados (stakeholders), como los pacientes y la comunidad. Cada vez es más reconocido que lo que ocurre en clínicas y hospitales es solo una porción del cuidado de la salud (Holden et al., 2020); y que el autocuidado por pacientes y no profesionales juega un papel crítico. Sin embargo, fueron pocos los estudios (15) que se enfocaron en esta población.

Enfoques que entienden el cuidado de la salud como un sistema complejo han demostrado ser más eficaces (Braithwaite, 2018); entre estos enfoques sobresalen los relacionados con la resiliencia de la organización/sistema. Sin embargo, este dominio ha sido escasamente abordado en la investigación, solo 9\% (7 artículos) para resiliencia. En estos estudios destaca la intención de estudiar un mayor número de interacciones que pueden influir la forma como se hace el trabajo ("work as done") en contextos complejos y el efecto que eso puede tener en los resultados (Bertoni et al., 2021; Jatobá et al., 2016).

\section{Falta de estudios sobre el análisis de incidentes de seguridad del paciente}

Como se mencionó en la introducción, en las dos últimas décadas la E/FH ha tenido un papel importante en materia de seguridad del paciente (Carayon, et al., 2014a; Hignett et al., 2013). Sin embargo, parece que eso no ha sucedido aún en el contexto de los sistemas de salud en América Latina, dado que en la revisión no fue posible identificar algún estudio dentro de este dominio. Una posible explicación para esto podría ser que la investigación de la seguridad del paciente es relativamente nueva en comparación con otros ámbitos e industrias (Hignett et al., 2013; Waterson, 2009). Otra posible explicación para esto es la insuficiente investigación y falta de reporte de incidentes de seguridad del paciente en la región. Por ejemplo, fue apenas en 2011 que la OMS comisionó el primer informe de seguridad del paciente en América Latina (IBEAS), que incluía un estudio específico de eventos adversos (WHO, 2011). A la fecha sigue siendo unos de los pocos que existen en América Latina. 


\section{Fortalezas y limitaciones del estudio}

Una de las fortalezas de este estudio es su sentido inclusivo, dado tres características: 1) la amplitud de las palabras clave utilizadas para la búsqueda; 2) la inclusión de estudios en inglés, español y portugués; y 3) la inclusión de estudios con metodologías y principios derivados de la $\mathrm{E} / \mathrm{FH}$, incluso si no estaba explícitamente dicho que se trataba de un estudio de E/FH. Al mismo tiempo, una limitación es que en la revisión no se incluyeron artículos de congresos, capítulos de libros o algunos otros documentos de la llamada literatura gris. De igual manera, el análisis de esta revisión podría expandirse para conocer, por un lado, las aportaciones de los estudios en cada uno de los dominios y, por otro lado, cuál ha sido el impacto de la investigación al interior de los sistemas de salud investigados.

\section{Conclusiones}

Esta revisión sistemática describe el estado de la investigación sobre E/HF en los sistemas de salud de América Latina. En general, los resultados sugieren la existencia de investigación en $\mathrm{E} / \mathrm{FH}$ en el sector de la salud en América Latina. Sin embargo, también se observa que existen aún dominios de la $\mathrm{E} / \mathrm{FH}$ poco explorados, a pesar de la urgencia por una cobertura de los servicios de salud más segura y efectiva.

En particular se observa un uso escaso de un enfoque de sistemas en la investigación. Es necesario destacar que mejoras más sostenibles en los sistemas de salud de América Latina demandan de la aplicación del enfoque de sistemas para el análisis y el diseño de múltiples interacciones en los sistemas de salud. Por otra parte, la investigación se ha realizado principalmente en Brasil y es prácticamente inexistente y/o nula en el resto de los países. Esta situación hace manifiesta la necesidad de incrementar el reconocimiento y la demanda del uso de la ergonomía en el sector salud.

Es importante destacar que el reciente llamado de la OMS para incorporar a la ergonomía como una estrategia para construir organizaciones de salud más seguras y resilientes es una excelente oportunidad para integrar la E/FH en los sistemas de salud, y a la vez un gran desafío para todos los académicos y profesionales de la ergonomía en la región. Como parte de ese reto, la Red Latinoamericana de Ergonomía y Factores Humanos en Sistemas de Salud (RELAESA) tiene como parte de sus objetivos crear una comunidad de práctica para poner en contacto las necesidades de las comunidades sanitarias con la experiencia de los profesionales e investigadores en $\mathrm{E} / \mathrm{FH}$, y con ello generar un mayor reconocimiento y demanda por la disciplina. El énfasis de esta comunidad de práctica es la promoción y aplicación de la ergonomía con una clara perspectiva de sistemas que ayude a conservar el bienestar físico y psicológico de pacientes y trabajadores de la salud. 


\section{Referencias}

Abdalla, D. R., Freitas, F. S. de, Matheus, J. P. C., Walsh, I. A. P. de, \& Bertoncello, D. (2014). Postural biomechanical risks for nursing workersRiscos biomecânicos posturais em trabalhadores de enfermagem. Fisioterapia Em Movimento, 27(3), 421-427. https://doi.org/10.1590/0103-5150.027.003.AO13

Aceves-González, C., Rodríguez, Y., Escobar-Galindo, C. M., Pérez, E., Gutiérrez-Moreno, B., Hignett, S., \& Lang, A. R. (2021). Frontiers in human factors: integrating human factors and ergonomics to improve safety and quality in Latin American healthcare systems. International Journal for Quality in Health Care, 33(Supplement_1), 45-50. https://doi.org/10.1093/intqhc/mzaa135

Alencar, M. do C. B. de, Schultze, V. M., \& Souza, S. D. de. (2010). Distúrbios osteomusculares e o trabalho dos que cuidam de idosos institucionalizados. Fisioterapia Em Movimento, 23(1 PG-63-72), 63-72. https://doi.org/10.1590/S0103-51502010000100006

Alexandre, N. M. C., Moraes, M. A. A. de, Corrêa Filho, H. R., \& Jorge, S. A. (2001). Evaluation of a program to reduce back pain in nursing personnel. Revista de Saúde Pública, 35(4 PG356-361), 356-361. http://www.scielosp.org/scielo.php?script=sci $\operatorname{arttext\& pid=So034-~}$ 89102001000400004\&lang=en

Andrade, L. de, Lynch, C., Carvalho, E., Rodrigues, C. G., Vissoci, J. R. N., Passos, G. F., Pietrobon, R., Nihei, O. K., \& de Barros Carvalho, M. D. (2014). System Dynamics Modeling in the Evaluation of Delays of Care in ST-Segment Elevation Myocardial Infarction Patients $\begin{array}{llllll}\text { within a Tiered Health System. PLoS ONE, 9(7), e103577. } & \text {. }\end{array}$ https://doi.org/10.1371/journal.pone.0103577

Barnes-Farrell, J. L., Davies-Schrils, K., McGonagle, A., Walsh, B., Milia, L. D., Fischer, F. M., Hobbs, B. B., Kaliterna, L., \& Tepas, D. (2008). What aspects of shiftwork influence off-shift well-being of healthcare workers? Applied Ergonomics, 39(5 PG-589-596), 589-596. https://doi.org/10.1016/j.apergo.2008.02.019

Barra, D. C. C., \& Dal Sasso, G. T. M. (2010). Mobile bedside technology: Computerized nursing processes in intensive care unit from ICNP 1.o . Texto e Contexto Enfermagem, 19(1 PG-5463), 54-63. https://doi.org/10.1590/s0104-07072010000100006

Barros, J. O., Gonçalves, R. M. A., Kaltner, R. P., \& Lancman, S. (2015). Matrix support strategies: The experience of two family health support centers (NASFs) in São Paulo, Brazil . Ciencia e Saude Coletiva, 20 (9 PG-2847-2856), 2847-2856. https://doi.org/10.1590/141381232015209.12232014

Bautista, L., Maradei, F., \& Pedraza, G. (2020). Usability test with medical personnel of a handgesture control techniques for surgical environment. International Journal on Interactive Design and Manufacturing (IJIDeM), 14(3), 1031-1040. https://doi.org/10.1007/s12008020-00690-9

Bernardes, M., Trzesniak, C., Trbovich, P., \& Mello, C. H. P. (2018). Applying human factors engineering methods for hazard identification and mitigation in the radiotherapy process. Safety Science, 109(PG-270-280), 270-280. https://doi.org/10.1016/j.ssci.2018.06.002

Bertoni, V. B., Saurin, T. A., Fogliatto, F. S., Falegnami, A., \& Patriarca, R. (2021). Monitor, anticipate, respond, and learn: Developing and interpreting a multilayer social network of resilience abilities. Saf. Sci., 136(PG-). https://doi.org/10.1016/j.ssci.2020.105148

Binda, J., Bianco, M. de F., \& Sousa, E. M. de. (2013). O trabalho dos agentes comunitários de saúde em evidência: uma análise com foco na atividade. Saúde e Sociedade, 22(2 PG-389402), 389-402. https://doi.org/10.1590/So104-12902013000200011 
Bitkina, O. V., Kim, H. K., \& Park, J. (2020). Usability and user experience of medical devices: An overview of the current state, analysis methodologies, and future challenges. International Journal of Industrial Ergonomics, 76(February). https://doi.org/10.1016/j.ergon.2020.102932

Bolis, I., \& Sznelwar, L. I. (2016). A case study of the implementation of an ergonomics improvement committee in a Brazilian hospital - Challenges and benefits. Applied Ergonomics, 53(PG-181-189), 181-189. https://doi.org/10.1016/j.apergo.2015.09.012

Bolis, I., Sznelwar, L. I., \& Silva, M. T. da. (2013). O trabalho de atendentes em atividades administrativas de um serviço ambulatorial: o serviço e as relações com pacientes e médicos. Gestão \& Produção, 2O(2), 481-493. https://doi.org/10.1590/S0104-530X2013000200016

Braithwaite, J. (2018). Changing how we think about healthcare improvement. BMJ (Online), 361, 1-5. https://doi.org/10.1136/bmj.k2014

Caivano, S., Ferreira, B. J., \& Domene, S. M. Á. (2014). Avaliação da usabilidade do Guia Alimentar Digital móvel segundo a percepção dos usuários. Ciência \& Saúde Coletiva, 19(5), 1437-1446. https://doi.org/10.1590/1413-81232014195.13932013

Callegaro, A. M., Caten, C. S. ten, Tanure, R. L. Z., Buss, A. S., Echeveste, M. E. S., \& Jung, C. F. (2016). Managing requirements for the development of a novel elbow rehabilitation device. Technological Forecasting and Social Change, 113(PG-404-411), 404-411. https://doi.org/10.1016/j.techfore.2016.07.027

Campos-Filho, A. S., Novaes, M. A., \& Gomes, A. S. (2018). The Mental Workload Evaluation in Visualizing Telehealth Indicators on Three-Dimensional Interface. Telemedicine and EHealth, 24(6), 442-448. https://doi.org/10.1089/tmj.2017.0127

Carayon, P., Wetterneck, T. B., Rivera-Rodriguez, a. J., Hundt, A. S., Hoonakker, P., Holden, R., \& Gurses, A. P. (2014a). Human factors systems approach to healthcare quality and patient safety. Applied Ergonomics, 45(1), 14-25. https://doi.org/10.1016/j.apergo.2013.04.023

Carayon, P., Xie, A., \& Kianfar, S. (2014b). Human factors and ergonomics as a Patient safety practice. BMJ Quality and Safety, 23(3), 196-205. https://doi.org/10.1136/bmjqs-2013$\underline{001812}$

Carmona, J. E., Higuerey, J. A., Gil, D., Castillo, M., \& Escalona, V. (2019). Physical and Mental Impact of Laparoscopic Sleeve Gastrectomy on the Surgeon: French vs. American Positions. A Randomized and Controlled Study. Obesity Surgery, 29(1), 137-142. https://doi.org/10.1007/s11695-018-3496-1

Carneiro, M. I. S., Oliveira, D. M. De, Maciel, A. B. D. R., Cardoso, A. C. D. A., Teichrieb, V., \& Monte-Silva, K. (2016). Applicability of a motor rehabilitation system in stroke victims. Fisioterapia Em Movimento, 29(4 PG-723-730), 723-730. https://doi.org/10.1590/19805918.029.004.0008

Carregaro, R. L., Toledo, A. M. de, Christofoletti, G., Oliveira, A. B. de, Cardoso, J. R., \& Padula, R. S. (2013). Association between work engagement and perceived exertion among healthcare workers. Fisioterapia Em Movimento, 26(3 PG-579-585), 579-585. https://doi.org/10.1590/S0103-51502013000300011

Carvalho, L. R. de, Domingues, A. N., \& Zem-Mascarenhas, S. H. (2018). Desenvolvimento de tecnologia digital educacional sobre monitoração da pressão intracraniana minimamente invasiva. Texto \& Contexto - Enfermagem, 26(4). https://doi.org/10.1590/010407072017000830017

Carvalho, L. R. de, Évora, Y. D. M., \& Zem-Mascarenhas, S. H. (2016). Assessment of the usability of a digital learning technology prototype for monitoring intracranial pressure. Revista Latino-Americana de Enfermagem, 24(PG-). https://doi.org/10.1590/1518-8345.1054.2777 
Catchpole, K., Bowie, P., Fouquet, S., Rivera, J., \& Hignett, S. (2021). Frontiers in human factors: embedding specialists in multi-disciplinary efforts to improve healthcare. International Journal for Quality in Health Care, 33(Supplement_1), 13-18. https://doi.org/10.1093/intqhc/mzaa108

Chapanis, A., \& Safren, M. (1960). Of misses and medicines. Journal of Chronic Diseases, 12, $103-408$.

Chughtai, S., \& Blanchet, K. (2017). Systems thinking in public health: A bibliographic contribution to a meta-narrative review. Health Policy and Planning, 32(4). https://doi.org/10.1093/heapol/czw159

Churruca, K., Pomare, C., Ellis, L. A., Long, J. C., \& Braithwaite, J. (2019). The influence of complexity: A bibliometric analysis of complexity science in healthcare. BMJ Open, 9(3). https://doi.org/10.1136/bmjopen-2018-027308

Chartered Institute of Ergonomics and Human Factors. (2018). Human Factors for Health and Social Care - White Paper.

Coelho, A. C. C., Souza-Machado, A., Leite, M., Almeida, P., Castro, L., Cruz, C. S., Stelmach, R., \& Cruz, Á. A. (2011). Manuseio de dispositivos inalatórios e controle da asma em asmáticos graves em um centro de referência em Salvador. Jornal Brasileiro de Pneumologia, 37(6), 720-728. https://doi.org/10.1590/S1806-37132011000600004

da Costa, F. M., de Barros, N. F., de Oliveira, H. C., \& Alexandre, N. M. C. (2020). Effects of an intervention program with health education and hatha yoga on the health of professionals with musculoskeletal symptoms. Revista Brasileira de Medicina Do Trabalho, 18(02), 114124. https://doi.org/10.47626/1679-4435-2020-492

de Almeida, S. R. W., Dal Sasso, G. T. M., \& Barra, D. C. C. (2016). Computerized nursing process in the Intensive Care Unit: Ergonomics and usability. Revista Da Escola de Enfermagem, 50(6 PG-996-1002), 996-1002. https://doi.org/10.1590/Soo80-623420160000700017

de Carvalho, E. A., Gomes, J. O., Jatobá, A., da Silva, M. F., \& de Carvalho, P. V. R. (2021). Employing resilience engineering in eliciting software requirements for complex systems: experiments with the functional resonance analysis method (FRAM). Cognition, Technology \& Work, 23(1), 65-83. https://doi.org/10.1007/s10111-019-00620-0

Del Valle Royas, A., \& Marziale, M. H. P. (2001). A situação de trabalho do pessoal de enfermagem no contexto de um hospital argentino: um estudo sob a ótica da ergonomia. Revista Latino-Americana de Enfermagem, 9(1 PG-102-108), 102-108. https://doi.org/10.1590/S0104-11692001000100015

Delgado, D., Aguilera, M. de los Á., Delgado, F., Cano, I., \& Ramírez, Ó. (2013). Calidad de Vida en el Trabajo y Condiciones de Trabajo, en Auxiliares de Esterilización. Ciencia \& Trabajo, 15(48), 148-151. https://doi.org/10.4067/So718-24492013000300007

Díaz Gutiérrez, C. D., González Portal, G., Espinosa Tejeda, N., Díaz Batista, R., \& Espinosa Tejeda, I. (2013). Trastornos músculo esquelético y ergonomía en estomatólogos del municipio Sancti Spíritus. 2011. Gaceta Médica Espirituana, 15(1 PG-75-82), 75-82. http://scielo.sld.cu/scielo.php?script=sci $\operatorname{arttext\& pid=S1608-}$

89212013000100010\&lang $=\mathrm{pt}$

Do Carmo Alonso, C. M., De Lima, A. N., Oggioni, B. D. M. P., Teixeira, M. R., Oliveira, E. P., Couto, M. C. V, \& Duarte, F. J. C. M. (2020). Contributions of activity ergonomics to the design of an electronic health record to support collaborative mental care of children and youth: Preliminary results. Work, 65(1 PG-187-194), 187-194. https://doi.org/10.3233/WOR-193048 
Duarte, N. S., \& Mauro, M. Y. C. (2010). Análise dos fatores de riscos ocupacionais do trabalho de enfermagem sob a ótica dos enfermeiros. Revista Brasileira de Saúde Ocupacional, 35(121 PG-157-167), 157-167. https://doi.org/10.1590/So303-76572010000100017

Dul, J., Bruder, R., Buckle, P., Carayon, P., Falzon, P., Marras, W. S., Wilson, J. R., \& van der Doelen, B. (2012). A strategy for human factors/ergonomics: developing the discipline and profession. Ergonomics, 55(4), 377-395. https://doi.org/10.1080/00140139.2012.661087

Feitosa-Beleza, C. M., De Oliveira Gouveia, M. T., Do Carmo Cruz Robazzi, M. L., Dias Torres, C. R., \& Viana De Azevedo, G. A. (2013). Occupational risks and health problems perceived by professional nursing in hospital unit. Ciencia y Enfermeria, 19(3 PG-73-82), 73-82. https://doi.org/10.4067/So717-95532013000300008

Fernandes, M. A., \& Marziale, M. H. P. (2014). Riscos ocupacionais e adoecimento de trabalhadores em saúde mental. Acta Paulista de Enfermagem, 27(6), 539-547. https://doi.org/10.1590/1982-0194201400088

Ferreira, D. S., Ramos, F. R. S., \& Teixeira, E. (2021). Aplicativo móvel para a práxis educativa de enfermeiros da estratégia saúde da família: ideação e prototipagem. Escola Anna Nery, 25(1 PG-). https://doi.org/10.1590/2177-9465-ean-2019-0329

Gama, L. N., \& Tavares, C. M. M. (2019). Development and evaluation of mobile application for the prevention of musculoskeletal risks in nursing work . Texto e Contexto Enfermagem, 28(PG-). https://doi.org/10.1590/1980-265X-TCE-2018-0214

Garbin, A. J. Í., Garbin, C. A. S., Diniz, D. G., \& Yarid, S. D. (2011). Dental students' knowledge of ergonomic postural requirements and their application during clinical care. European Journal of Dental Education, 15(1), 31-35. https://doi.org/10.1111/j.16000579.2010.00629.x

Grebin, S. Z., Echeveste, M. E. S., Magnago, P. F., Tanure, R. L. Z., \& Pulgati, F. H. (2018). Estratégia de análise para avaliação da usabilidade de dispositivos médicos na percepção do usuário: um estudo com pacientes em tratamento de hemodiálise. Cadernos de Saúde Pública, 34(8 PG-). https://doi.org/10.1590/0102-311x00074417

Grossi, L. M., Pisa, I. T., \& Marin, H. de F. (2014). Oncoaudit: desenvolvimento e avaliação de aplicativo para enfermeiros auditores. Acta Paulista de Enfermagem, 27(2 PG-179-185), 179-185. https://doi.org/10.1590/1982-0194201400031

Guedes, R. M. de A., Lima, F. de P. A., \& Assunção, A. Á. (2005). O programa de qualidade no setor hospitalar e as atividades reais da enfermagem: o caso da medicação. Ciência \& Saúde Coletiva, 1O(4), 1063-1074. https://doi.org/10.1590/S1413-81232005000400029

Herrera-Saray, P., Peláez-Ballestas, I., Ramos-Lira, L., Sánchez-Monroy, D., \& Burgos-Vargas, R. (2013). Problemas con el uso de sillas de ruedas y otras ayudas técnicas y barreras sociales a las que se enfrentan las personas que las utilizan. Estudio cualitativo desde la perspectiva de la ergonomía en personas discapacitadas por enfermedades reumáticas y ot. Reumatología Clínica, 9(1), 24-30. https://doi.org/10.1016/j.reuma.2012.05.010

Hignett, S., Carayon, P., Buckle, P., \& Catchpole, K. (2013). State of science: human factors and ergonomics in healthcare. Ergonomics, 56(10), 1491-1503. https://doi.org/10.1080/00140139.2013.822932

Holden, R. J., Cornet, V. P., \& Valdez, R. S. (2020). Patient ergonomics: 10-year mapping review of patient-centered human factors. Applied Ergonomics, 82. https://doi.org/10.1016/j.apergo.2019.102972

International Ergonomics Association. (2000). International Ergonomics Asociation. Definition of Ergonomics. https://iea.cc/ 
Isosaki, M., Cardoso, E., Glina, D. M. R., Pustiglione, M., \& Rocha, L. E. (2011). Intervenção nas situações de trabalho em um serviço de nutrição hospitalar e repercussões nos sintomas osteomusculares. Revista de Nutrição, 24(3 PG-449-462), 449-462. https://doi.org/10.1590/S1415-52732011000300008

Jatoba, A., da Cunha, A. M., Vidal, M. C. R., Burns, C. M., \& de Carvalho, P. V. R. (2019). Contributions from cognitive engineering to requirements specifications for complex sociotechnical systems: A case study in the context of healthcare in Brazil. Human Factors and Ergonomics In Manufacturing, 29(1 PG-63-77), 63-77. https://doi.org/10.1002/hfm.20758

Jatobá, A, Bellas, H. C., Bonfatti, R., Burns, C. M., Vidal, M. C. R., \& de Carvalho, P. V. R. (2016). Designing for patient risk assessment in primary health care: a case study for ergonomic work analysis. Cognition, Technology and Work, $18(1$ PG-215-231), 215-231. https://doi.org/10.1007/s10111-015-0355-X

Jatobá, Alessandro, Bellas, H. C., Bulhões, B., Koster, I., Arcuri, R., \& de Carvalho, P. V. R. (2020). Assessing community health workers' conditions for delivering care to patients in low-income communities. Applied Ergonomics, 82(PG-), 102944. https://doi.org/10.1016/j.apergo.2019.102944

Jorge, M. S. B., Costa, L. S. P., Carvalho, M. R. R., Mamede, R. S. de B., Morais, J. B. de, \& Paula, M. L. de. (2020). Mobile web application for use in the Extended Family Health and Primary Care Center: content and usability validation. Revista CEFAC, 22(3 PG-). https://doi.org/10.1590/1982-0216/20202233519

Kataife, E. D., Said, S., Braun, J., Roche, T. R., Rössler, J., Kaserer, A., Spahn, D. R., Mileo, F. G., \& Tscholl, D. W. (2020). The Haemostasis Traffic Light, a user-centred coagulation management tool for acute bleeding situations: a simulation-based randomised dual-centre trial. Anaesthesia, PG-. https://doi.org/10.1111/anae.15314

Kohn, L., Corrigan, J., \& Donaldson, M. (2000). To Err Is Human: Building a Safer Health System. National Academies Press. https://doi.org/10.17226/9728

Lacerda, T. C., von Wangenheim, C. G., von Wangenheim, A., \& Giuliano, I. (2014). Does the use of structured reporting improve usability? A comparative evaluation of the usability of two approaches for findings reporting in a large-scale telecardiology context. Journal of Biomedical Informatics, 52(PG-222-230), 222-230. https://doi.org/10.1016/j.jbi.2014.07.002

Lima, C. J. M. de, Coelho, R. A., Medeiros, M. S., Kubrusly, M., Marçal, E., \& Peixoto Júnior, A. A. (2019). Development and Validation of a Mobile Application for the Teaching of Electrocardiogram. Revista Brasileira de Educação Médica, 43(1 suppl 1), 157-165. https://doi.org/10.1590/1981-5271v43suplemento1-20190164.ing

Lima, M., Jeane Pinto Chaves, B., Da Silva Lima, V., Silva, P. E., Correia Sobral Soares, N. S., \& Beserra da Costa Santos, I. (2018). Riscos ocupacionais em profissionais de enfermagem de centros de material e esterilização. Revista Cuidarte, 9(3), 1-8. https://doi.org/10.15649/cuidarte.v9i3.544

Lopes, J. P., Dias, T. M. R., Carvalho, D. B. F., Oliveira, J. F. De, Cavalcante, R. B., \& Oliveira, V. C. De. (2019). Evaluation of digital vaccine card in nursing practice in vaccination room. Revista Latino-Americana de Enfermagem, 27(PG-). https://doi.org/10.1590/15188345.3058.3225

Manchi-Zuloeta, F. R., Chávez-Rimache, L. K., Chacón-Uscamaita, P. R., Chumpitaz-Cerrate, V., \& Rodriguez-Vargas, M. C. (2019). Relación entre las posturas de trabajo y síntomas musculoesqueléticos en estudiantes de odontología en Lima. Revista Habanera de Ciencias 
Médicas,

$18(5$

PG-730-740),

$730-740$.

http://scielo.sld.cu/scielo.php?script=sci arttext\&pid=S1729519X2019000500730\&lang $=\mathrm{pt}$

Marques, A. D. B., Moreira, T. M. M., Jorge, T. V., Rabelo, S. M. S., Carvalho, R. E. F. L. de, \& Felipe, G. F. (2020). Usability of a mobile application on diabetic foot self-care. Revista Brasileira de Enfermagem, 73(4 PG-). https://doi.org/10.1590/0034-7167-2018-0862

Melo, C. M. C. da S., Delgado Filho, A. J. F., Oliveira, E. R. de, Araújo, A. A. de, Cavalcanti, H. G. de O., Melo, C. M. C. da S., Bushatsky, M., Sanches, L. M. P., \& Barros, M. B. S. C. (2020). Development and Assessment of an Application for Primary Care for Users with Diabetes Mellitus. Aquichan, 2O(2 PG-). https://doi.org/10.5294/aqui.2020.20.2.6

Mendes, D. P., Oliveira, M. M. de, Matos, V. G. de, Mazoni, M. B., \& Moraes, G. F. de S. (2012). Do prescrito ao real: a gestão individual e coletiva dos trabalhadores de enfermagem frente ao risco de acidente de trabalho. Gestão \& Produção, 19(4), 885-892. https://doi.org/10.1590/S0104-530X2012000400016

Mogollón, I. C. M., Maroto, M. M., \& González, A. R. Á. (2020). Patient safety culture in nursing training. Revista Cubana de Enfermeria, 36(2 PG-1-14), 1-14. https://www.scopus.com/inward/record.uri?eid=2-s2.085090734148\&partnerID $=40 \&$ md $5=$ of 7777 b677afceo99153da 5 ebf2254fo

Montalvo-Prieto, A. A., Cortés-Múnera, Y. M., \& Rojas-López, M. C. (2015). Riesgo ergonómico asociado a sintomatología musculoesquelética en personal de enfermería. Hacia La Promoción de La Salud, $20(2 \quad$ PG-132-146), $\quad$ 132-146. https://doi.org/10.17151/hpsal.2015.20.2.11

Montoya Díaz, M. del C., Palucci Marziale, M. H., do Carmo Cruz Robazzi, M. L., \& Taubert de Freitas, F. C. (2010). Lesiones osteomusculares en trabajadores de un hospital mexicano y la ocurrencia del ausentismo. Ciencia y Enfermería, $16(2$ PG-35-46), 35-46. https://doi.org/10.4067/So717-95532010000200005

Moreira, R. F. C., Moriguchi, C. S., Carnaz, L., Foltran, F. A., Silva, L. C. C. B., \& Coury, H. J. C. G. (2020). Effects of a workplace exercise program on physical capacity and lower back symptoms in hospital nursing assistants: a randomized controlled trial. International Archives of Occupational and Environmental Health, PG-. https://doi.org/10.1007/s00420020-01572-Z

Muñoz, C. R., Estupiñan, J. C. M., Borrero, A. J. C., Panameño, C. L. M., García, J. J. L., Huertas, J. V, Daza, M. M. D., \& Valencia, M. J. H. (2019). Ergonomic risk in the surgical staff of different hospital institutions in the city of Santiago de Cali, 2018 . Archivos Venezolanos de $\begin{array}{lllll}\text { Farmacologia } y & \text { Terapeutica, } & 38(4 \quad \text { PG-509-513), } & \text { 509-513. }\end{array}$ https://www.scopus.com/inward/record.uri?eid=2-s2.08507857743.39\&partnerID $=40 \& \mathrm{md} 5=7$ cd912a2f3b783b04c935oebde1 56121

Nery, D., Toledo, A. M., Oliveira Júnior, S., Taciro, C., \& Carregaro, R. (2013). Análise de parâmetros funcionais relacionados aos fatores de risco ocupacionais da atividade de enfermeiros de UTI. Fisioterapia $e$ Pesquisa, $20(1$ PG-76-82), 76-82. https://doi.org/10.1590/S1809-29502013000100013

Organisation for Economic Cooperation and Development/The World Bank. (2020). Health at a Glance: Latin America and the Caribbean 2020. In Health at a Glance: Latin America and the Caribbean 2020. OECD Publishing. https://doi.org/10.1787/6089164f-en

Oliveira Dantas, F. F., \& de Lima, K. C. (2015). The relationship between physical load and musculoskeletal complaints among Brazilian dentists. Applied Ergonomics, 47(PG-93-98), 93-98. https://doi.org/10.1016/j.apergo.2014.09.003 
Oliveira, N. B. de, \& Peres, H. H. C. (2015). Evaluation of the functional performance and technical quality of an Electronic Documentation System of the Nursing Process. Revista Latino-Americana de Enfermagem, $23(2 \quad$ PG-242-249), 242-249. https://doi.org/10.1590/0104-1169.3562.2548

Padrini-Andrade, L., Balda, R., Areco, K., Bandiera-Paiva, P., Nunes, M. do V., Marba, S., Carvalho, W., Rugolo, L., Almeida, J., Procianoy, R., Duarte, J. L., Rego, M. A., Ferreira, D. M., Alves Filho, N., Guinsburg, R., Diniz, E. M., Santos, J. P., Testoni, D., Silva, N. M., ... Bomfim, O. (2018). Evaluation of usability of a neonatal health information system according to the user's perception. Revista Paulista de Pediatria, 37(1 PG-90-96), 90-96. https://doi.org/10.1590/1984-0462/;2019;37;1;00019

Pinto, F. do M., \& Zambroni-de-Souza, P. C. (2015). A atividade de trabalho de motoristas de ambulância sob o ponto de vista ergológico. Revista Brasileira de Saúde Ocupacional, 4O(131), 49-58. https://doi.org/10.1590/0303-7657000076113

Plack, M. M., Goldman, E. F., Scott, A. R., Pintz, C., Herrmann, D., Kline, K., Thompson, T., \& Brundage, S. B. (2018). Systems Thinking and Systems-Based Practice Across the Health Professions: An Inquiry Into Definitions, Teaching Practices, and Assessment. Teaching and Learning in Medicine, 3O(3). https://doi.org/10.1080/10401334.2017.1398654

Ransolin, N., Saurin, T. A., \& Formoso, C. T. (2020). Integrated modelling of built environment and functional requirements: Implications for resilience. Applied Ergonomics, 88(PG-), 103154. https://doi.org/10.1016/j.apergo.2020.103154

Ribeiro Custódio, R. A., Trzesniak, C., Pinto Ribeiro Miranda, R., Donda Angelini, G. H., Bordón, J. S., Santos Vieira, L. C., \& Pereira Mello, C. H. (2019). Applying Human Factors Engineering Methods for Risk Assessment of a Neonatal Incubator. Journal of Healthcare Engineering, (PG-). https://doi.org/10.1155/2019/8589727

Righi, A. W., \& Saurin, T. A. (2015). Complex socio-technical systems: Characterization and management guidelines. Applied Ergonomics, 5O(PG-19-30), 19-30. https://doi.org/10.1016/j.apergo.2015.02.003

Rosso, C. B., \& Saurin, T. A. (2018). The joint use of resilience engineering and lean production for work system design: A study in healthcare. Applied Ergonomics, 71(PG-45-56), 45-56. https://doi.org/10.1016/j.apergo.2018.04.004

Ruggeri, B. F. F., Voci, S. M., Borges, C. A., \& Slater, B. (2013). Assessment of the usability of a nutritional epidemiology computerized system. Revista Brasileira de Epidemiologia, 16(4 PG-966-975), 966-975. https://doi.org/10.1590/S1415-790X2013000400016

Safren, M., \& Chapanis, A. (1960). A critical incident study of hospital medication errors. Hospitals, 1(34), 32-34.

Santiago, R. F., Andrade, E. M. L. R., Mendes, I. A. C., Viana, M. C. A., \& Nery, I. S. (2020). Avaliação de objeto virtual de aprendizagem sobre pré-natal para adolescentes grávidas na atenção básica. Acta Paulista de Enfermagem, 33(PG-). https://doi.org/10.37689/actaape/2020a00063

Scott, P. (2008). Global inequality, and the challenge for ergonomics to take a more dynamic role to redress the situation. Applied Ergonomics, 39(4), 495-499. https://doi.org/10.1016/j.apergo.2008.02.014

Shimabukuro, V. G. P., Alexandre, N. M. C., Coluci, M. Z. O., Rosecrance, J. C., \& Gallani, M. C. J. B. (2012). Validity and Reliability of a Job Factors Questionnaire Related to the Work Tasks of Physical Therapists. International Journal of Occupational Safety and Ergonomics, 18(1), 15-26. https://doi.org/10.1080/10803548.2012.11076911 
Soares, G. B., Siqueira, C. E., Estrada-Martínez, L., Garbin, C. A. S., \& Garbin, A. J. I. (2019). Musculoskeletal Disorders among Brazilian Dentists in São Paulo. Revista Odonto Ciencia, 33(1 PG-33-39), 33-39. https://doi.org/10.15448/1980-6523.2018.1.29699

Soares, M. (2006). Ergonomics in Latin America: Background, trends and challenges. Applied Ergonomics, 37(4), 555-561. https://doi.org/10.1016/j.apergo.2006.04.014

Souza, M. L. de M., Meneghini, A. C., Ferraz, É., Vianna, E. O., \& Borges, M. C. (2009). Técnica e compreensão do uso dos dispositivos inalatórios em pacientes com asma ou DPOC. Jornal Brasileiro de Pneumologia, 35(9), 824-831. https://doi.org/10.1590/S180637132009000900002

Sznelwar, L. I., Lancman, S., Wu, M. J., Alvarinho, E., \& Santos, M. dos. (2004). Análise do trabalho e serviço de limpeza hospitalar: contribuições da ergonomia e da psicodinâmica do trabalho. Production, 14(3 PG-45-57), 45-57. https://doi.org/10.1590/So103$\underline{6} 5132004000300006$

Sznelwar, L., Silva, M. T., \& Mascia, F. L. (2008). Working in public health services in Brazil: The relationship between different rationalities. Applied Ergonomics, 39(4), 500-508. https://doi.org/10.1016/j.apergo.2008.02.008

Thatcher, A., \& Todd, A. (2019). HFE in Underdeveloped Countries. In R. Roscoe, E. Chiou, \& A. Wooldridge (Eds.), Advancing Diversity, Inclusion, and Social Justice Through Human Systems Engineering (pp. 31-50). https://doi.org/10.1201/9780429425905-3

Tottoli, C. R., Toledo, A. M. de, Silva, N. C. e, Araújo, W. N. de, Souza, R. da N., \& Carregaro, R. L. (2019). Profissionais da saúde que atuam em ambiente hospitalar têm alta prevalência de fadiga e dorsalgia: estudo transversal. Fisioterapia e Pesquisa, 26(1 PG-91-100), 91-100. https://doi.org/10.1590/1809-2950/18032926012019

Venegas, T. C. E., \& Cochachin, C. J. E. (2019). Nivel de conocimiento sobre riesgos ergonómicos en relación a síntomas de trastornos músculo esqueléticos en personal sanitario. Revista de La Asociación Española de Especialistas En Medicina Del Trabajo, 28(2 PG-126-135), 126135 . $\underline{62552019000200005 \& l a n g=p t}$

Wachs, P., Saurin, T. A., Righi, A. W., \& Wears, R. L. (2016). Resilience skills as emergent phenomena: A study of emergency departments in Brazil and the United States. Applied Ergonomics, 56(PG-227-237), 227-237. https://doi.org/10.1016/j.apergo.2016.02.012

Waterson, P. (2009). A critical review of the systems approach within patient safety research. Ergonomics, 52(10), 1185-1195. https://doi.org/10.1080/00140130903042782

Waterson, P. (2011). World War II and other historical influences on the formation of the Ergonomics Research Society. Ergonomics, 54(12), 1111-1129. https://doi.org/10.1080/00140139.2011.622796

World Health Organization. (2011). IBEAS: a pioneer study on patient safety in Latin America. Towards safer hospital care.

World Health Organization. (2021). Global patient safety action plan 2021 - 2030. Towards Eliminating Avoidable Harm in Health Care, 3(1), 1-109.

Wilson, J. R. (2014). Fundamentals of systems ergonomics/human factors. Applied Ergonomics, 45(1), 5-13. https://doi.org/10.1016/j.apergo.2013.03.021 


\section{Anexo}

Tabla A1. Panorama de los estudios en ergonomía/factores humanos por dominio.

\begin{tabular}{|c|c|c|c|c|}
\hline Dominio & Reference & Revista / Disciplina & Población de estudio & $\begin{array}{l}\text { Palabra asociada a } \\
\text { E/FH }\end{array}$ \\
\hline \multirow{26}{*}{ USABILIDAD } & Souza et al. (2009) & Jornal Brasileiro de Pneumologia & Pacientes con enfermedades respiratorias & Uso de dispositivos \\
\hline & Barra \& Dal Sasso (2010) & Texto \& Contexto - Enfermagem & $\begin{array}{l}\text { Enfermeras, educadores de cuidado } \\
\text { intensivo y programadores de sistemas }\end{array}$ & $\begin{array}{l}\text { Ergonomía - } \\
\text { usabilidad }\end{array}$ \\
\hline & Ruggeri et al. (2013) & Revista Brasileira de Epidemiologia & $\begin{array}{l}\text { Profesionales de tecnología de la } \\
\text { información (TI) }\end{array}$ & Usabilidad \\
\hline & Grossi et al. (2014) & Acta Paulista de Enfermagem & Enfermeras y profesionales de TI & Usabilidad \\
\hline & Caivano et al. (2014) & Ciencia \& saude coletiva & Usuarios & Usabilidad \\
\hline & Lacerda et al. (2014) & Journal of Biomedical Informatics & Usuarios & Usabilidad \\
\hline & Oliveira \& Peres (2015) & Revista Latino-Americana de Enfermagem & $\begin{array}{l}\text { Profesionales de TI, maestros de } \\
\text { enfermería y enfermeras }\end{array}$ & Usabilidad \\
\hline & De Almeida et al. (2016) & Revista da Escola de Enfermagem & $\begin{array}{l}\text { Enfermeras, maestros y programadores } \\
\text { de TI }\end{array}$ & Usabilidad \\
\hline & Carneiro et al. (2016) & Fisioterapia em Movimento & $\begin{array}{l}\text { Pacientes en rehabilitación debido a } \\
\text { accidentes cerebrovasculares }\end{array}$ & Usabilidad \\
\hline & Carvalho et al. (2016) & Revista Latino-Americana de Enfermagem & $\begin{array}{l}\text { Expertos en Interacción Humano- } \\
\text { Computadora }\end{array}$ & $\begin{array}{l}\text { Interacción Humano- } \\
\text { computadora }\end{array}$ \\
\hline & Campos Filho et al. (2018) & Telemedicine and health & Profesionales en tele-salud & Usabilidad \\
\hline & Carvalho et al., (2018) & Texto Contexto Enfermagen & Expertos en informática & Usabilidad \\
\hline & Grebin et al. (2018) & Cadernos de Saúde Pública & $\begin{array}{l}\text { Ingenieros clínicos, enfermeras, } \\
\text { nefrólogo, ergónomo, un paciente }\end{array}$ & Usabilidad \\
\hline & Padrini-Andrade et al. (2018) & Revista Paulista de Pediatría & Médicos y enfermeras & Usabilidad \\
\hline & Ribeiro Custódio et al. (2019) & Journal of Healthcare Engineering & $\begin{array}{l}\text { Enfermeras, técnicos y estudiantes de } \\
\text { enfermería }\end{array}$ & Usabilidad \\
\hline & Lima et al. (2019) & Revista Brasileira de Educação Médica & Estudiantes y maestros de cardiología & Usabilidad \\
\hline & Lopes et al. (2019) & Revista Latino-Americana de Enfermagem & Usuarios y profesionales de la salud & Usabilidad \\
\hline & Gama \& Tavares (2019) & Texto \& Contexto Enfermagem & Enfermeras y profesionales de TI & Usabilidad \\
\hline & Santiago et al. (2020) & Acta Paulista de Enfermagem & Enfermeras y profesionales de TI & Usabilidad \\
\hline & Melo et al. (2020) & Aquichan & Enfermeras & Usabilidad \\
\hline & Bautista et al., (2020) & $\begin{array}{l}\text { International Journal on Interactive Design and } \\
\text { Manufacturing }\end{array}$ & Estudiantes y cirujanos ortopédicos & Usabilidad \\
\hline & Marques et al. (2020) & Revista Brasileira de Enfermagen & Usuarios & Usabilidad \\
\hline & Jorge et al. (2020) & $\begin{array}{l}\text { Revista CEFAC - Speech, Language, Hearing, } \\
\text { Sciences and Education Journal }\end{array}$ & Profesionales de la salud & Usabilidad \\
\hline & Do Carmo Alonso et al. (2020) & Work & $\begin{array}{l}\text { Personal de salud y personal de un centro } \\
\text { de investigación }\end{array}$ & Ergonomía \\
\hline & Kataife et al. (2020) & Anaesthesia & Médicos residentes y anestesiólogos & $\begin{array}{l}\text { Diseño Centrado en el } \\
\text { Usuario }\end{array}$ \\
\hline & (Ferreira et al. (2021) & Escola Anna Nery Revista de Enfermagen & Enfermeras & Usabilidad \\
\hline
\end{tabular}


EID Ergonomía, Investigación y Desarrollo, 3(2), 2021, 10-27

ISSN 2452-4859

\begin{tabular}{|c|c|c|c|c|}
\hline Dominio & Reference & Revista / Disciplina & Población de estudio & $\begin{array}{l}\text { Palabra asociada a } \\
\text { E/FH }\end{array}$ \\
\hline \multirow{24}{*}{$\begin{array}{l}\text { DESEMPEÑO Y } \\
\text { SEGURIDAD } \\
\text { DE LOS } \\
\text { TRABAJADORES }\end{array}$} & Alexandre et al. (2001) & Revista Saúde Pública & Auxiliares de enfermería & Ergonomía \\
\hline & Barnes-Farrell et al. (2008) & Applied Ergonomics & Personal de salud & Bienestar \\
\hline & Montoya Díaz et al. (2010) & Ciencia y enfermería & Trabajadores de hospital & Ergonomía \\
\hline & Alencar et al. (2010) & Fisioterapia em movimento & Cuidadores de personas adultas mayores & Ergonomía \\
\hline & Duarte \& Mauro (2010) & Revista Brasileira de Saude Ocupacional & Jefas de enfermería & Ergonomía \\
\hline & Garbin et al. (2011) & European Journal of Dental Education & Estudiantes de odontología & Ergonomía \\
\hline & Isosaki et al., (2011) & Revista de Nutrição & Personal de salud & Ergonomía \\
\hline & Mendes et al. (2012) & Gestão \& Produção & Enfermeras & Ergonomía \\
\hline & Shimabukuro et al., (2012) & $\begin{array}{l}\text { International Journal of Occupational Safety and } \\
\text { Ergonomics }\end{array}$ & fisioterapeutas & Ergonomía \\
\hline & Feitosa et al. (2013) & Ciencia y Enfermería & Enfermeras & Ergonomía \\
\hline & Delgado et al. (2013) & Ciencia y Trabajo & Auxiliares de esterilización & Ergonomía \\
\hline & Nery et al. (2013) & Fisioterapia e Pesquisa & Enfermeras de cuidado intensivo & Ergonomía \\
\hline & Carregaro et al. (2013) & Fisioterapia em Movimento & Personal femenino de salud & Ergonomía \\
\hline & Díaz et al. (2013) & Gaceta Médica Espirituana & Estomatólogos & Ergonomía \\
\hline & Binda et al. (2013) & Saúde e Sociedade & Agentes de salud comunitaria & Ergología \\
\hline & Fernandes \& Marziale (2014) & Acta Paulista de Enfermagem & $\begin{array}{l}\text { Trabajadores de la salud, con diferentes } \\
\text { profesiones }\end{array}$ & Ergonomía \\
\hline & Abdalla et al. (2014) & Fisioterapia em Movimento & Técnicos de enfermería & Ergonomía \\
\hline & Oliveira Dantas \& de Lima (2015) & Applied Ergonomics & Dentistas & Ergonomía \\
\hline & (Barros et al., (2015) & Ciencia \& saúde coletiva & $\begin{array}{l}\text { Trabajadores de la salud, con diferentes } \\
\text { profesiones }\end{array}$ & Ergonomía \\
\hline & Montalvo Prieto et al. (2015) & Hacia la Promoción de la Salud & Enfermeras & Ergonomía \\
\hline & Pinto \& Zambroni-de-Souza (2015) & Revista Brasileira de Saúde Ocupacional & Conductores de ambulancia & Ergonomía \\
\hline & Lima et al. (2018) & Revista Cuidarte & Enfermeras & Ergonomía \\
\hline & Soares et al. (2019) & Revista Odonto Ciencia & Dentistas & Ergonomía \\
\hline & Muñoz et al. (2019) & $\begin{array}{l}\text { Archivos Venezolanos de Farmacología y } \\
\text { Terapéutica }\end{array}$ & Personal de cirugía & Ergonomía \\
\hline Dominio & Reference & Revista / Disciplina & Población de estudio & $\begin{array}{c}\text { Palabra asociada a } \\
\text { E/FH }\end{array}$ \\
\hline \multirow{9}{*}{$\begin{array}{l}\text { DESEMPEÑOY } \\
\text { SEGURIDAD } \\
\text { DE LOS } \\
\text { TRABAJADORES } \\
\text { (continuación) }\end{array}$} & Tottoli et al. (2019) & Fisioterapia e Pesquisa & $\begin{array}{l}\text { Trabajadores de la salud, con diferentes } \\
\text { profesiones }\end{array}$ & Ergonomía \\
\hline & Venegas \& Cochachin, (2019) & $\begin{array}{l}\text { Revista de la Asociación Española de Especialistas } \\
\text { en Medicina del Trabajo }\end{array}$ & Enfermeras y personal técnico & Ergonomía \\
\hline & Manchi-Zuloeta et al. (2019) & Revista Habanera de Ciencias Médicas & Estudiantes de Odontología & Ergonomía \\
\hline & Carmona et al. (2019) & Obesity Surgery & Cirujanos laparoscopia & Ergonomía \\
\hline & Jatoba et al. (2020) & Applied Ergonomics & Personal de salud comunitaria & $\begin{array}{l}\text { Condiciones de } \\
\text { trabajo }\end{array}$ \\
\hline & da Costa et al. (2020) & Revista Brasileira de Medicina do Trabalho & Personal de administrativo y de apoyo & Ergonomía \\
\hline & Mogollón et al. (2020) & Revista Cubana de Enfermería & Estudiantes & $\begin{array}{l}\text { Seguridad del } \\
\text { paciente }\end{array}$ \\
\hline & Moreira et al. (2020) & $\begin{array}{l}\text { International Archives of Occupational and } \\
\text { Environmental Health }\end{array}$ & Asistentes de enfermería & $\begin{array}{l}\text { Síntomas musculo } \\
\text { esqueléticos }\end{array}$ \\
\hline & Sznelwar et al. (2004) & Revista Produção & Personal de salud (sin caracterizar) & Ergonomía \\
\hline
\end{tabular}




\begin{tabular}{|c|c|c|c|c|}
\hline \multirow{8}{*}{$\begin{array}{l}\text { DISEÑO DE } \\
\text { SISTEMAS }\end{array}$} & Guedes et al. (2005) & Ciencia \& saúde coletiva & Enfermeras & Ergonomía \\
\hline & Sznelwar et al. (2008) & Applied Ergonomics & Personal de salud de primera línea & Ergonomía \\
\hline & Herrera-Saray et al. (2013) & Reumatología Clínica & Pacientes usuarios de sillas de ruedas & Ergonomía \\
\hline & Andrade et al. (2014) & Plos ONE & Médicos, enfermeras y administrativos & $\begin{array}{l}\text { Modelado de sistemas } \\
\text { dinámicos }\end{array}$ \\
\hline & Jatoba et al. (2019) & $\begin{array}{l}\text { Human Factors and Ergonomics in } \\
\text { Manufacturing \& Service Industries }\end{array}$ & Médicos, enfermeras y administrativos & $\begin{array}{l}\text { Sistemas complejos - } \\
\text { modelado de sistemas }\end{array}$ \\
\hline & Righi \& Saurin (2015) & Applied Ergonomics & Médicos y enfermeras & $\begin{array}{l}\text { Ergonomía - diseño - } \\
\text { sistemas complejos }\end{array}$ \\
\hline & Bolis \& Sznelwar (2016) & Applied Ergonomics & $\begin{array}{l}\text { Enfermeras, médicos, fisioterapeutas, } \\
\text { administrativos y personal de salud y } \\
\text { seguridad }\end{array}$ & Ergonomía \\
\hline & Jatobá et al.(2016) & Cognition, Technology and Work & $\begin{array}{l}\text { Enfermeras, camilleros, personal } \\
\text { administrativo y directivo }\end{array}$ & $\begin{array}{l}\text { Ergonomía - } \\
\text { evaluación de riesgo }\end{array}$ \\
\hline \multirow{3}{*}{$\begin{array}{l}\text { RESILIENCIA } \\
\text { ORGANIZACIONAL/ } \\
\text { DE SISTEMAS }\end{array}$} & Del Valle Royas \& Marziale (2001) & Revista Latino Americana de Emfermagen & Enfermeras & Ergonomía \\
\hline & Bolis et al. (2013) & Gestão \& Produção & Personal de salud y pacientes & Ergonomía \\
\hline & Wachs et al. (2016) & Applied ergonomics & $\begin{array}{l}\text { Médicos, enfermeras y técnicos } \\
\text { enfermería }\end{array}$ & Resiliencia \\
\hline Dominio & Reference & Revista / Disciplina & Población de estudio & $\begin{array}{c}\text { Palabra asociada a } \\
\text { E/FH } \\
\end{array}$ \\
\hline \multirow{4}{*}{$\begin{array}{l}\text { RESILIENCIA } \\
\text { ORGANIZACIONAL/ } \\
\text { DE SISTEMAS } \\
\text { (continuación) }\end{array}$} & Rosso \& Saurin (2018) & Applied ergonomics & $\begin{array}{l}\text { Médicos, enfermeros, técnicos y } \\
\text { trabajadores administrativos }\end{array}$ & $\begin{array}{l}\text { Ingeniería de } \\
\text { resiliencia }\end{array}$ \\
\hline & Ransolin et al. (2020) & Applied ergonomics & $\begin{array}{l}\text { Médicos, enfermeras, directivos, } \\
\text { familiares, pacientes, técnicos de } \\
\text { enfermería, personal de limpieza }\end{array}$ & $\begin{array}{l}\text { Ingeniería de } \\
\text { resiliencia }\end{array}$ \\
\hline & de Carvalho et al. (2021) & Cognition, Technology \& Work & Ingenieros de software & $\begin{array}{l}\text { Ingeniería de } \\
\text { resiliencia }\end{array}$ \\
\hline & Bertoni et al. (2021) & Safety Science & $\begin{array}{l}\text { Enfermeras, técnicos de enfermería, } \\
\text { profesionales aliados, y médicos }\end{array}$ & $\begin{array}{l}\text { Ingeniería de } \\
\text { resiliencia }\end{array}$ \\
\hline \multicolumn{5}{|l|}{ MIXTO } \\
\hline $\begin{array}{l}\text { Usabilidad y desempeño y } \\
\text { Seguridad de los } \\
\text { trabajadores }\end{array}$ & Coelho et al. (2011) & Jornal Brasileiro de Pneumologia & Pacientes & Uso de dispositivos \\
\hline $\begin{array}{l}\text { Usabilidad y Diseño de } \\
\text { sistemas }\end{array}$ & Callegaro et al. (2016) & Technological Forecasting and Social Change & $\begin{array}{l}\text { Pacientes, estudiantes del área de } \\
\text { desarrollo de productos, expertos y } \\
\text { representantes de empresas, médicos y } \\
\text { fisioterapeutas. }\end{array}$ & Ergonomía \\
\hline $\begin{array}{l}\text { Usabilidad y Resiliencia } \\
\text { organizacional/ de } \\
\text { Sistemas }\end{array}$ & Bernardes et al. (2018) & Safety Science & Profesionales de Radioterapia & Ergonomía \\
\hline
\end{tabular}

